\title{
MORFOLOGIA DE AGREGADOS DO SOLO AVALIADA POR MEIO DE ANÁLISE DE IMAGE NS(1)
}

\author{
N. OLSZEVSKI (2), L. M. COSTA ${ }^{(3)}$, E. I. FERNANDES FILHO(3), \\ H. A. RUIZ(3), R. C. ALVARENGA ${ }^{(4)} \&$ J . C. CRUZ ${ }^{(4)}$
}

\begin{abstract}
RESUMO
O uso intensivo do solo, aliado a condições inadequadas de manejo, concorre para a deterioração de suas propriedades físi cas e isso se deve, principalmente, às modificações em sua estrutura. Uma vez que as mudanças estruturais influem diretamente na morfologia dos agregados, considera-se fundamental a procura de novos métodos de estudos macromorfológicos para permitir o acompanhamento das modificações dessa característica nos solos cultivados. Sendo assim, este trabalho teve por objetivo adaptar e testar um método digital de análise macromorfológica qualitativa de agregados, visando detectar modificações morfológi cas causadas pela ação antrópica. Para tal estudo, foram escolhidos cinco sistemas de preparo do solo: plantio di reto, escarificador, grade aradora, arado de discos e arado de ai vecas. Realizou-se o estudo da forma e do tamanho das diferentes classes de agregados de um Latossolo Vermel ho distrófico típico, em amostras coletadas em 1998, após a aquisição de imagens com o uso de um scanner (HP 6100C com capacidade de resolução ótica de 1.200 dpi) e o posterior processamento dessas imagens pelo programa de computador denominado UTHSCSA Image Tool. Este programa fornece índices sobre os agregados, tais como: arredondamento, compacidade e alongamento. $O$ índice arredondamento é dependente da medida de perímetro (rugosidade externa) e os índices compacidade e alongamento são dependentes da medida do comprimento do maior eixo (arestas). De maneira geral, os sistemas de manejo utilizados por três anos consecutivos não promoveram diferenças morfológicas dos agregados do Latossolo Vermelho textura argilosa para as variáveis analisadas. A análise de imagens mostrou-se sensível à detecção de mudanças na morfologia dos agregados do solo, sendo bastante promissora como uma nova ferramenta nos estudos da estrutura do solo.
\end{abstract}

Termos de indexação: análise macromorfológica de agregados, sistemas de manejo, alongamento, arredondamento, compacidade.

\footnotetext{
(1) Parte da Tese de Mestrado do primeiro autor, apresentada ao Departamento de Solos da Universidade Federal de Viçosa - UFV. Recebido para publicação em junho de 2002 e aprovado em setembro de 2004.

(2) Professora do Departamento de Zootecnia da Faculdade de Imperatriz - FACIMP. Av. Prudente de Morais s/n, CEP 65900-000 I mperatriz (MA). E-mail: nelci @facimp.edu.br

(3) Professor do Departamento de Solos da Universidade Federal de Viçosa - UFV. Av. PH Rolfs s/n, CEP $36570-000$ Viçosa (MG). Bolsista do CNPq.E-mails: liovando@solos.ufv.br; el pidio@ufv.br; hruiz@ufv.br

(4) Pesquisador do Centro Naicional de Pesquisa de Milho e Sorgo da Embrapa. Caixa Postal 151, CEP 35701-970 Sete Lagoas (MG). E-mails: ramon@cnpms.embrapa.br; zecarlos@cnpms.embrapa.br
} 


\title{
SUMMARY: MORPHOLOGY OF SOIL AGGREGATES EVALUATED BY IMAGE ANALYSIS
}

\begin{abstract}
The intensive soil use together with inadequate management practices contribute to thedeterioration of soil physical properties, mainly dueto modifications in thesoil structure pattern. Once the structural changes could affect the morphology aggregates in different ways, thesearch for new methods of studying soil macro-morphol ogical characteristics must be prioritized to allow the observation of modified characteristics in cultivated soils. Therefore, this study was carried out to adapt and test a digital method for a qualitative macro-morphological analysis of soil aggregates of Rodhic Haplustox in samples taken in 1998, aiming to detect morphol ogical modifications caused by human activities. From an original set of eleven, fivereplicated treatments werese ected: no till, cinzel pl ow, heavy disk harrow, disk plow and chisel plow, carried out annually. The shape of the different soil aggregates was from images obtained using a scanner (HP 6100C with 1200 dpi optical resolution) which were then processed by the computer program UTHSCSA I mage Tool. This program furnishes values of different aggregateindexes: roundness, compactness and lengthiness. The roundness index depends on the perimeter measurement (external roughness) and thecompactness and lengthiness indexes depend on themeasurement of the length of the longest axle (long edges). Generally, the management systems used during three consecutive years did not modify the aggregate morphology of the clayey Rodhic Haplustox for the analyzed variables. I mage analysis sensitively detected alterations in soil aggregate morphology promising considerable usefulness as a novel tool for on soil structurestudies.
\end{abstract}

Index terms: macro-morphol ogical analysis of aggregates, management systems lengthiness, roundness, compactness.

\section{NTRODUÇÃO}

O uso intensivo do solo, aliado a condições inadequadas demanejo, concorre para a deterioração de suas propriedades físicas e isso se deve, principalmente, às modificações em sua estrutura. Para Brewer (1976), a estrutura representa a própria constituição física do solo, expressa pelo tamanho, pela forma e pela distribuição ou pelo arranjamento dos espaços vazios e das partículas sólidas do solo, sejam elas primárias ou secundárias. Fernandes (1982) e Dadalto et al. (1989) concluíram que os agregados mostram diferenças quanto a tamanho, forma, estabilidadee adesão entresi equealterações nas condições naturais do solo por sistemas de manejo podem al terar o tamanho, a distribuição e a estabilidade desses agregados

Em estudos de Sedimentologia, a forma e o arredondamento dos grãos deareia e dos seixos têm sido usados, desde há muito tempo, para decifrar histórias de depósitos sedimentares (Suguio, 1973). Ainda de acordo com esse autor, uma descrição da forma geométrica de partículas envolve normalmente vários conceitos relacionados. De um Iado, destacam-se os fatores de forma, que dependem dos comprimentos dos eixos principais perpendiculares entre si e, de outro, os fatores de angularidade ou arredondamento das partículas. Os dois conceitos são importantes nos estudos de sedimentos de diferentes maneiras. A forma ou as relações de comprimento dos eixos controlam parcial mente o comportamento dos seixos durante o transporte e a deposição, enquanto o arredondamento, ou angularidade, reflete a distância e o rigor do transporte. Para isso, necessita-se de um processo simples e objetivo que permita expressar numericamentea forma dos grãos, não somente para fins descritivos, mas também para execução de estudos quantitativos de vários fatores envolvidos na evolução, até à forma final da partícula ou do fragmento.

Um dos métodos utilizados em Sedimentologia é o uso de tabelas de comparação visual, para determinar os graus de arredondamento e esfericidade de areias (Suguio, 1973). A esfericidade está relacionada com a proporção de comprimentolargura das imagens das partículas, enquanto o arredondamento é expresso pela curvatura das arestas das imagens.

Uma vez que as mudanças estruturais influem diretamente na morfologia dos agregados, considera-se fundamental a procura de novos métodos de estudos macromorfol ógicos para permitir o acompanhamento das modi ficações dessa característica nos sol os cultivados. Dessa forma, a utilização de instrumentos práticos, mais sensíveis para a predição da sustentabilidade do uso desse recurso natural, seria de interesse. 
O programa de computador denominado UTHSCSA I mage Tool (Wilcox et al., 1997) foi desenvolvido pelo Departamento de Diagnose Dentária no Centro de Ciências da Saúde da Universidade do Texas em San Antonio no Texas (EUA) para estudos na área odontológica. Como o programa tem a capacidade de processar e analisar imagens e, posteriormente, fornecer valores de diferentes unidades de medida, foi utilizado para o estudo de agregados de solo.

Assim, o objetivo deste trabal ho foi, a partir de um programa computacional, adaptar etestar esse método digital de análise macromorfológica qual itativa de agregados dos solos, visando detectar modificações morfológicas causadas pela ação antrópica, em amostras de um Latossol o Vermel ho distrófico típico.

\section{MATERIAL E MÉTODOS}

O experimento avaliado foi instalado no Centro Nacional de Pesquisa de Milho e Sorgo (CNPMS) Embrapa, localizado no município de Sete Lagoas (MG). Para testar a influência no rendimento da cultura do milho, o experimento foi instalado no ano agrícola de 1994/95, em Latossolo Vermelho distróficotípico textura argilosa, constando de cinco tratamentos de preparo do solo. Os tratamentos foram compostos de grade aradora $(G)$, arado de $\operatorname{discos}(D)$, arado de aivecas (A), escarificador (E), plantio direto (PD) e da associação dos arados com a grade pesada, cada um em um ano agrícola. O delineamento experimental utilizado foi o de blocos casual izados, com três repetições.

A col eta das amostras de sol o foi real izada no mês de outubro de 1998. Foram abertas trincheiras com dimensões de 0,60 × 0,60 × 0,60 ma 0,30 m da linha de plantio em cada unidade experimental. A amostragem foi efetuada em quatro profundidades: 0 a $0,075,0,075$ a 0,150, 0,150 a 0,225 e 0,225 a $0,300 \mathrm{~m}$, com o uso de uma pá reta, sendo o solo destorroado manualmente no campo, aplicando-se leve pressão para causar o mínimo de mudanças em sua estrutura original, sendo, a seguir, acondicionado em frasco de plástico rígido.

Para o estudo da forma dos agregados, $100 \mathrm{~g}$ de solo seco foram passados em um conjunto de peneiras com mal has de $2,1,0,5,0,25$ e 0,105 mm, dispostas em um vibrador horizontal, durante cinco minutos, evel oci dade de trabal ho do aparel ho na escala cinco, quando este oferece opções na faixa de zero a dez.

O estudo dos agregados foi realizado após a aquisição de suas imagens com o uso de um scanner (HP 6100C, com capacidade de resolução ótica de $1.200 \mathrm{dpi}$ ). As imagens foram processadas por meio do programa decomputador denominado UTHSCSA
Image Tool (Wilcox et al., 1997). Para isso, uma pequena amostra de cada frasco foi retirada com o uso da espátula, espalhada sobre o scanner, e os agregados separados manualmente, um a um, com o auxílio de estilete e de lupa. A resolução utilizada foi de $600 \mathrm{dpi}$ para os agregados provenientes das classes: $2,00-1,00,1,00-0,50$ e 0,50-0,210 mm, e de $1.200 \mathrm{dpi}$, para a classe 0,210-0,105 mm.

As variáveis analisadas nos agregados foram: área, perímetro, comprimento do maior eixo e do menor eixo, grau dearredondamento, al ongamento, diâmetro de Feret e compacidade. Essas variáveis fornecem informações sobre o tamanho, forma e rugosidade dos agregados, servindo de base para comparar diferentes sistemas de manejo do solo no que diz respeito à maior ou menor agressividade à estrutura dosolo. A seguir, são definidas as variáveis analisadas:

Área (Ar): corresponde ao número de pixels no polígono.

Perímetro (Pm): comprimento da projeção do limite exterior do agregado.

Comprimento do maior eixo (CME): comprimento de uma linha traçada na maior distância no agregado.

Comprimento do menor eixo: comprimento de uma linha traçada perpendicularmente ao maior eixo do agregado.

Alongamento: relação entre o comprimento do menor eixo e o comprimento do maior eixo. O resultado será um val or entre 0 e 1 . Quanto maior o valor, menor será a diferença entre os comprimentos dos eixos perpendiculares e menor o alongamento.

Arredondamento (Ard): medida dependente da rugosidade da superfície externa do agregado (perímetro). Fornecerá o resultado entre 0 e 1 e, quanto maior o val or, maior o grau de arredondamento. É calculado a partir da fórmula.

$$
\mathrm{Ard}=(4 \pi \mathrm{Ar}) / \mathrm{Pm}^{2}
$$

Diâmetro de Feret (DF): o diâmetro de um círculo com a mesma área do objeto. É calculado a partir da fórmula:

$$
\mathrm{DM}=\sqrt{(4 \mathrm{Ar} / \pi)}
$$

Compacidade (Cmp): fornece uma medida da circularidade do objeto, sendo dependente da medida do comprimento do maior eixo. Varia de 0 a 1 e, se for igual a 1, oagregadoé perfeitamente circular. ́́ calculado a partir da fórmula:

$$
\mathrm{Cmp}=\sqrt{(4 \mathrm{Ar} / \pi)} / \mathrm{CME}
$$


Os resultados obtidos foram submeti dos à análise de variância, utilizando-se o Sistema para Análises Estatísticas e Genéticas (SAEG), da Central de Processamento de Dados da UFV (Ribeiro J únior, 2001), realizada para cada profundidade, desdobrando-se os graus de liberdade para tratamentos (sistemas de manejo) mediante os seguintes contrastes ortogonais: $\mathrm{Cl}=4 \mathrm{PD}$ $(E+G+D+A), C 2=3 E-(G+D+A), C 3=2 G-$ $(D+A)$ e $C 4=D-A$. Consideram-se tratamentos diferentes quando significativos a $5 \%$ pelo teste $F$. Utilizou-se a palavra - tendência - para expressar diferenças estatísticas no intervalo entre 5 e $20 \%$, pelo teste $F$.

\section{RESULTADOS E DISCUSSÃO}

Para a classe de agregados com diâmetro de 2,00 a 1,00 mm, de maneira geral, não houve diferença entre os sistemas de preparo do sol o e pr ofundidades de amostragem para os val ores de área e perímetro e dos índices (Quadro 1). Para a variável área dos agregados, na profundidade de 0,000 a 0,075 m, o plantio direto apresentou tendência a maiores valores em comparação com os demais sistemas de manejo. Resultado semel hante foi observado para os sistemas de manejo com o uso da grade em relação ao comportamento dos arados de discos e de aiveca em conjunto. Tal resultado pode ser considerado coerente, visto que se partiu de um sistema que causa menor dano à estrutura do solo, no caso o plantio direto, atéo arado de discos, quecausa maior revolvimento, com conseqüente quebra e pulverização dos agregados.

Ainda para a variável área na profundidade de $0-0,075 \mathrm{~m}$ da classe de diâmetro de 1,00 a $0,50 \mathrm{~mm}$ (Quadro 2), o comportamento foi semel hante, exceto para o caso do escarificador, e, na classe de 0,50-0,21 mm (Quadro 3), o plantio direto mostrou tendência de maior ocorrência de agregados com área maior em relação aos implementos.

Acredita-se que sistemas de manejo que promovam menor revolvimento do solo devam apresentar maiores valores para a variável perímetro, graças à maior proporção de agregados de tamanho e rugosidade externa maiores. De maneira geral, o resultado observado não refletiu o resultado esperado.

Supõe-se também que sistemas que provoquem menor revolvimento do solo devam apresentar menores valores para a variável alongamento, em razão da menor quebra e posterior tendência do agregado à circularidade. Para a classe de diâmetro de 2,00-1,00 mm (Quadro 1), na profundidade de 0,000-0,075 m, o sistema de plantio direto mostrou o resultado esperado, o mesmo acontecendo na profundidade de 0,075-0,150 m, quando se compara o comportamento da grade com o dos arados em conjunto e com o do arado de discos com o do arado de aiveca, ou seja, em ordem crescente de agressividade à estrutura, encontram-se menores valores de alongamento. O mesmo foi observado para a classe de 1,00 a 0,50 mm (Quadro 2), exceto para o caso do arado de aivecas. Também na classe de diâmetro de 0,21 a 0,105 mm (Quadro 4), o plantio direto apresentou menores val ores de al ongamento na camada superficial $(0,000$ a 0,075 m).

Também para o índice arredondamento, os sistemas de manejo que provocam menor revolvimento do solo devem apresentar menores valores para a variável arredondamento, pois esteé dependente da medida de perímetro. Logo, maiores valores de arredondamento deverão ser encontrados para os agregados provenientes de sistemas de manejo mais agressivos à estrutura, pois estes tenderão a perder a rugosidade da superfície externa, diminuindo, assim, o valor do perímetro. De maneira geral, não foram observadas diferenças significativas entre as classes de diâmetro e profundidades de amostragem, com exceção da classe de diâmetro de 0,50 a 0,21 mm (Quadro 3) na camada superficial, em que o plantio direto apresentou menor val or para oíndice. Para a classe de 0,21 a 0,105 mm (Quadro 4) da camada superficial, o comportamento foi contrário ao esperado, pois mostrou tendência de aumento dos valores de arredondamento, quando se compara o resultado do escarificador com o da grade e o dos arados conjuntamente, bem como o comportamento da grade com o dos arados.

A variável compaci dade deveapresentar menores valores nos sistemas de manejo menos agressivos. Como descrito anteriormente, esta variável fornece uma medida da rugosidade do agregado, ou seja, quanto mais arestado, maior ovalor da compacidade. O observado para as classes de agregados com diâmetro de 2,00 a 1,00 mm (Quadro 1), 1,00 a 0,50 mm (Quadro 2) e 0,21 a 0,105 mm (Quadro 4) está de acordo com o esperado, ou seja, o plantio direto, sistema considerado menos agressivo, apresentou os menores valores para o índice.

Foram detectadas apenas pequenas alterações nos índices entre os diferentes sistemas de preparo do solo e, ou profundidades de amostragem, resultado que pode ser explicado de acordo com a classe de solo e com o tempo de condução do experimento, uma vez que se trata de um $L$ atossolo, ou seja, um solo bastante intemperizado e com estrutura granular considerada estável e homogênea. 
Quadro 1. Valores de área, perímetro, alongamento, arredondamento, diâmetro de Feret e compacidade, considerando o método de preparo e a profundidade de amostragem, e valores dos contrastes ortogonais para a classe de agregados de 2,00 a 1,00 mm de diâmetro

\begin{tabular}{|c|c|c|c|c|}
\hline \multirow{2}{*}{ Método de preparo } & \multicolumn{4}{|c|}{ Profundidade (m) } \\
\hline & 0,000 a 0,075 & 0,075 a 0,150 & 0,150 a 0,225 & 0,225 a 0,300 \\
\hline & \multicolumn{4}{|c|}{ Área, mm² } \\
\hline $\begin{array}{l}\text { Plantio direto (PD) } \\
\text { Escarificador (E) } \\
\text { Grade (G) } \\
\text { Disco (D) } \\
\text { Aiveca (A) } \\
\text { C1 } \\
\text { C2 } \\
\text { C3 } \\
\text { C4 }\end{array}$ & $\begin{array}{l}2,826 \\
2,807 \\
2,824 \\
2,019 \\
2,538 \\
1,115^{\#} \\
1,039^{\#} \\
1,091^{*} \\
-0,519^{\#}\end{array}$ & $\begin{array}{c}3,103 \\
3,197 \\
3,109 \\
2,705 \\
3,294 \\
\text { ns } \\
\text { ns } \\
\text { ns } \\
-0,588^{\#}\end{array}$ & $\begin{array}{l}2,698 \\
3,177 \\
2,824 \\
4,087 \\
2,684 \\
\text { ns } \\
\text { ns } \\
\text { ns } \\
1,402^{\#}\end{array}$ & $\begin{array}{l}2,587 \\
2,721 \\
3,197 \\
2,810 \\
2,906 \\
-1,285^{\#} \\
-0,749 \\
0,677^{\#} \\
-\end{array}$ \\
\hline $\mathrm{C} 4$ & \multicolumn{4}{|c|}{ Perímetro, mm } \\
\hline $\begin{array}{l}\text { Plantio direto (PD) } \\
\text { Escarificador (E) } \\
\text { Grade (G) } \\
\text { Disco (D) } \\
\text { Aiveca (A) } \\
\text { C1 } \\
\text { C2 } \\
\text { C3 } \\
\text { C4 }\end{array}$ & $\begin{array}{c}8,140 \\
7,937 \\
8,239 \\
6,290 \\
7,461 \\
\text { ns } \\
\text { ns } \\
2,726^{\#} \\
-1,170^{\#}\end{array}$ & $\begin{array}{c}8,476 \\
8,497 \\
8,509 \\
7,425 \\
8,618 \\
\text { ns } \\
\text { ns } \\
\text { ns } \\
-1,192^{\#}\end{array}$ & $\begin{array}{c}7,553 \\
8,894 \\
7,796 \\
9,806 \\
7,225 \\
\text { ns } \\
\text { ns } \\
\text { ns } \\
2,581^{\#}\end{array}$ & $\begin{array}{c}7,487 \\
7,660 \\
8,986 \\
7,832 \\
7,544 \\
\text { ns } \\
\text { ns } \\
2,595^{*} \\
\text { ns }\end{array}$ \\
\hline $\mathrm{C} 4$ & \multicolumn{4}{|c|}{ Alongamento } \\
\hline $\begin{array}{l}\text { Plantio direto (PD) } \\
\text { Escarificador (E) } \\
\text { Grade (G) } \\
\text { Disco (D) } \\
\text { Aiveca (A) } \\
\text { C1 } \\
\text { C2 } \\
\text { C3 } \\
\text { C4 }\end{array}$ & $\begin{array}{c}0,741 \\
0,757 \\
0,753 \\
0,759 \\
0,763 \\
-0,069 * \\
\text { ns } \\
\text { ns } \\
\text { ns }\end{array}$ & $\begin{array}{l}0,754 \\
0,744 \\
0,737 \\
0,747 \\
0,759 \\
\text { ns } \\
\text { ns } \\
-0,032^{*} \\
-0,011^{\#}\end{array}$ & $\begin{array}{c}0,749 \\
0,750 \\
0,752 \\
0,745 \\
0,748 \\
\text { ns } \\
\text { ns } \\
\text { ns } \\
\text { ns }\end{array}$ & $\begin{array}{l}0,749 \\
0,758 \\
0,747 \\
0,746 \\
0,741 \\
\text { ns } \\
0,039 * \\
\text { ns } \\
\text { ns }\end{array}$ \\
\hline & \multicolumn{4}{|c|}{ Arredondamento } \\
\hline $\begin{array}{l}\text { Plantio direto (PD) } \\
\text { Escarificador (E) } \\
\text { Grade (G) } \\
\text { Disco (D) } \\
\text { Aiveca (A) } \\
\text { C1 } \\
\text { C2 } \\
\text { C3 } \\
\text { C4 }\end{array}$ & $\begin{array}{l}0,542 \\
0,568 \\
0,540 \\
0,637 \\
0,571 \\
\text { ns } \\
\text { ns } \\
\text { ns } \\
\text { ns }\end{array}$ & $\begin{array}{c}0,552 \\
0,572 \\
0,563 \\
0,609 \\
0,553 \\
\text { ns } \\
\text { ns } \\
\text { ns } \\
-0,066 \#\end{array}$ & $\begin{array}{c}0,581 \\
0,519 \\
0,581 \\
0,567 \\
0,633 \\
\text { ns } \\
\text { ns } \\
\text { ns } \\
\text { ns }\end{array}$ & $\begin{array}{c}0,588 \\
0,581 \\
0,510 \\
0,576 \\
0,626 \\
\text { ns } \\
\text { ns } \\
-0,182^{*} \\
\text { ns }\end{array}$ \\
\hline & \multicolumn{4}{|c|}{ Diâmetro de F eret, mm } \\
\hline $\begin{array}{l}\text { Plantio direto (PD) } \\
\text { Escarificador (E) } \\
\text { Grade (G) } \\
\text { Disco (D) } \\
\text { Aiveca (A) } \\
\text { C1 } \\
\text { C2 } \\
\text { C3 } \\
\text { C4 }\end{array}$ & $\begin{array}{l}1,853 \\
1,854 \\
1,857 \\
1,579 \\
1,760 \\
0,363^{\#} \\
0,367^{\#} \\
0,374^{*} \\
-0,181^{\#}\end{array}$ & $\begin{array}{c}1,936 \\
1,968 \\
1,949 \\
1,821 \\
1,998 \\
\text { ns } \\
\text { ns } \\
\text { ns } \\
-0,177^{\#}\end{array}$ & $\begin{array}{c}1,797 \\
1,963 \\
1,862 \\
1,873 \\
1,806 \\
\text { ns } \\
\text { ns } \\
\text { ns } \\
\text { ns }\end{array}$ & $\begin{array}{c}1,779 \\
1,826 \\
1,978 \\
1,856 \\
1,878 \\
-0,422^{\#} \\
\mathrm{~ns} \\
0,222^{\#} \\
\mathrm{~ns}\end{array}$ \\
\hline C4 & \multicolumn{4}{|c|}{ Compacidade } \\
\hline $\begin{array}{l}\text { Plantio direto (PD) } \\
\text { Escarificador (E) } \\
\text { Grade (G) } \\
\text { Disco (D) } \\
\text { Aiveca (A) } \\
\text { C1 } \\
\text { C2 } \\
\text { C3 } \\
\text { C4 }\end{array}$ & $\begin{array}{c}0,805 \\
0,819 \\
0,812 \\
0,825 \\
0,825 \\
-0,062 * \\
\text { ns } \\
-0,025^{\#} \\
\text { ns }\end{array}$ & $\begin{array}{l}0,810 \\
0,813 \\
0,807 \\
0,812 \\
0,816 \\
\text { ns } \\
\text { ns } \\
\text { ns } \\
\text { ns }\end{array}$ & $\begin{array}{l}0,809 \\
0,812 \\
0,817 \\
0,810 \\
0,817 \\
\text { ns } \\
\text { ns } \\
\text { ns } \\
\text { ns }\end{array}$ & $\begin{array}{l}0,816 \\
0,820 \\
0,811 \\
0,812 \\
0,813 \\
\text { ns } \\
0,023^{*} \\
\text { ns } \\
\text { ns }\end{array}$ \\
\hline
\end{tabular}

$\mathrm{C} 1=4 \mathrm{PD}-(\mathrm{E}+\mathrm{G}+\mathrm{D}+\mathrm{A}) ; \mathrm{C} 2=3 \mathrm{E}-(\mathrm{G}+\mathrm{D}+\mathrm{A}) ; \mathrm{C} 3=2 \mathrm{G}-(\mathrm{D}+\mathrm{A}) ; \mathrm{C} 4=\mathrm{DA}$.

ns:não-significativo; ${ }^{*} \mathrm{e}$ : significativos a 20 e $5 \%$, respectivamente, pelo teste $F$. 
Quadro 2. Valores de área, perímetro, alongamento, arredondamento, diâmetro de Feret e compacidade, considerando o método de preparo e a profundidade de amostragem, e valores dos contrastes ortogonais para a classe de agregados de 1,00 a $0,50 \mathrm{~mm}$

\begin{tabular}{|c|c|c|c|c|}
\hline \multirow{2}{*}{ Método de preparo } & \multicolumn{4}{|c|}{ Profundidade (m) } \\
\hline & 0,000 a 0,075 & 0,075 a 0,150 & 0,150 a 0,225 & 0,225 a 0,300 \\
\hline & \multicolumn{4}{|c|}{ Área, $\mathrm{mm}^{2}$} \\
\hline $\begin{array}{l}\text { Plantio direto (PD) } \\
\text { Escarificador (E) } \\
\text { Grade (G) } \\
\text { Disco (D) } \\
\text { Aiveca (A) } \\
\text { C1 } \\
\text { C2 } \\
\text { C3 } \\
\text { C4 }\end{array}$ & $\begin{array}{c}0,685 \\
0,592 \\
0,667 \\
0,582 \\
0,642 \\
0,258^{\#} \\
-0,113^{\#} \\
0,109^{*} \\
-0,059^{\#}\end{array}$ & $\begin{array}{c}0,628 \\
0,698 \\
0,786 \\
0,683 \\
0,726 \\
\text { ns } \\
\text { ns } \\
\text { ns } \\
-0,043^{\#}\end{array}$ & $\begin{array}{c}0,633 \\
0,595 \\
0,726 \\
0,683 \\
0,667 \\
\text { ns } \\
\text { ns } \\
\text { ns } \\
0,016^{\#}\end{array}$ & $\begin{array}{c}0,573 \\
0,727 \\
0,773 \\
0,650 \\
0,617 \\
-0,475 \# \\
\text { ns } \\
0,278 \# \\
\text { ns }\end{array}$ \\
\hline C4 & \multicolumn{4}{|c|}{ Perímetro, $\mathrm{mm}$} \\
\hline $\begin{array}{l}\text { Plantio direto (PD) } \\
\text { Escarificador (E) } \\
\text { Grade (G) } \\
\text { Disco (D) } \\
\text { Aiveca (A) } \\
\text { C1 } \\
\text { C2 } \\
\text { C3 } \\
\text { C4 }\end{array}$ & $\begin{array}{c}3,520 \\
3,356 \\
3,416 \\
3,239 \\
3,505 \\
\text { ns } \\
\text { ns } \\
0,087 \# \\
-0,266 \#\end{array}$ & $\begin{array}{c}3,353 \\
3,629 \\
3,920 \\
3,599 \\
3,701 \\
\text { ns } \\
\text { ns } \\
\text { ns } \\
-0,101^{\#}\end{array}$ & $\begin{array}{c}3,383 \\
3,280 \\
3,708 \\
3,576 \\
3,541 \\
\text { ns } \\
\text { ns } \\
\text { ns } \\
0,035 \#\end{array}$ & $\begin{array}{l}3,151 \\
3,690 \\
3,802 \\
3,543 \\
3,324 \\
\text { ns } \\
\text { ns } \\
0,737 * \\
0,219 \#\end{array}$ \\
\hline C4 & \multicolumn{4}{|c|}{ Alongamento } \\
\hline $\begin{array}{l}\text { Plantio direto (PD) } \\
\text { Escarificador (E) } \\
\text { Grade (G) } \\
\text { Disco (D) } \\
\text { Aiveca (A) } \\
\text { C1 } \\
\text { C2 } \\
\text { C3 } \\
\text { C4 }\end{array}$ & $\begin{array}{c}0,729 \\
0,735 \\
0,757 \\
0,758 \\
0,743 \\
-0,077 * \\
\text { ns } \\
\text { ns } \\
\text { ns }\end{array}$ & $\begin{array}{c}0,737 \\
0,736 \\
0,733 \\
0,742 \\
0,736 \\
\text { ns } \\
\text { ns } \\
-0,010^{*} \\
0,005^{\#}\end{array}$ & $\begin{array}{l}0,737 \\
0,749 \\
0,735 \\
0,745 \\
0,747 \\
\text { ns } \\
\text { ns } \\
\text { ns } \\
\text { ns }\end{array}$ & $\begin{array}{l}0,745 \\
0,739 \\
0,729 \\
0,740 \\
0,748 \\
\text { ns } \\
0,003^{*} \\
\text { ns } \\
\text { ns }\end{array}$ \\
\hline $\begin{array}{l}\text { C3 } \\
\text { C4 }\end{array}$ & \multicolumn{4}{|c|}{ Arredondamento } \\
\hline $\begin{array}{l}\text { Plantio direto (PD) } \\
\text { Escarificador (E) } \\
\text { Grade (G) } \\
\text { Disco (D) } \\
\text { Aiveca (A) } \\
\text { C1 } \\
\text { C2 } \\
\text { C3 } \\
\text { C4 }\end{array}$ & $\begin{array}{l}0,682 \\
0,666 \\
0,699 \\
0,694 \\
0,656 \\
\text { ns } \\
\text { ns } \\
\text { ns } \\
\text { ns }\end{array}$ & $\begin{array}{l}0,690 \\
0,664 \\
0,645 \\
0,660 \\
0,662 \\
\text { ns } \\
\text { ns } \\
\text { ns } \\
\text { ns }\end{array}$ & $\begin{array}{c}0,688 \\
0,688 \\
0,664 \\
0,665 \\
0,665 \\
\text { ns } \\
0,068^{\#} \\
\text { ns } \\
\text { ns }\end{array}$ & $\begin{array}{c}0,711 \\
0,669 \\
0,666 \\
0,652 \\
0,690 \\
\text { ns } \\
\text { ns } \\
-0,009 * \\
\text { ns }\end{array}$ \\
\hline $\begin{array}{l}\text { C3 } \\
\text { C4 }\end{array}$ & \multicolumn{4}{|c|}{ Diâmetro de Feret, mm } \\
\hline $\begin{array}{l}\text { Plantio direto (PD) } \\
\text { Escarificador (E) } \\
\text { Grade (G) } \\
\text { Disco (D) } \\
\text { Aiveca (A) } \\
\text { C1 } \\
\text { C2 } \\
\text { C3 } \\
\text { C4 }\end{array}$ & $\begin{array}{c}0,910 \\
0,850 \\
0,896 \\
0,844 \\
0,881 \\
0,169 * \\
-0,069^{\#} \\
0,065^{*} \\
-0,036^{\#}\end{array}$ & $\begin{array}{c}0,872 \\
0,919 \\
0,977 \\
0,911 \\
0,939 \\
\text { ns } \\
\text { ns } \\
\text { ns } \\
-0,028^{\#}\end{array}$ & $\begin{array}{c}0,879 \\
0,849 \\
0,938 \\
0,910 \\
0,901 \\
\text { ns } \\
-0,201 \# \\
\text { ns } \\
\text { ns }\end{array}$ & $\begin{array}{c}0,833 \\
0,941 \\
0,970 \\
0,887 \\
0,867 \\
-0,332^{\#} \\
\text { ns } \\
0,186^{\#} \\
\text { ns }\end{array}$ \\
\hline $\begin{array}{l}C 3 \\
\text { C4 }\end{array}$ & \multicolumn{4}{|c|}{ Compacidade } \\
\hline $\begin{array}{l}\text { Plantio direto (PD) } \\
\text { Escarificador (E) } \\
\text { Grade (G) } \\
\text { Disco (D) } \\
\text { Aiveca (A) } \\
\text { C1 } \\
\text { C2 } \\
\text { C3 } \\
\text { C4 }\end{array}$ & $\begin{array}{c}0,824 \\
0,827 \\
0,842 \\
0,843 \\
0,831 \\
-0,045^{*} \\
\text { ns } \\
0,011^{\#} \\
\text { ns }\end{array}$ & $\begin{array}{c}0,830 \\
0,826 \\
0,819 \\
0,828 \\
0,824 \\
\text { ns } \\
\text { ns } \\
\text { ns } \\
\text { ns }\end{array}$ & $\begin{array}{c}0,831 \\
0,839 \\
0,825 \\
0,831 \\
0,833 \\
\text { ns } \\
\text { ns } \\
\text { ns } \\
\text { ns }\end{array}$ & $\begin{array}{c}0,840 \\
0,826 \\
0,823 \\
0,827 \\
0,839 \\
\text { ns } \\
-0,009 * \\
\text { ns } \\
\text { ns }\end{array}$ \\
\hline
\end{tabular}

$\mathrm{C} 1=4 \mathrm{PD}-(\mathrm{E}+\mathrm{G}+\mathrm{D}+\mathrm{A}) ; \mathrm{C} 2=3 \mathrm{E}-(\mathrm{G}+\mathrm{D}+\mathrm{A}) ; \mathrm{C} 3=2 \mathrm{G}-(\mathrm{D}+\mathrm{A}) ; \mathrm{C} 4=\mathrm{DA}$

ns:não-significativo; ${ }^{*} \mathrm{e}^{*}$ : significativos a 20 e $5 \%$, respectivamente, pelo teste $F$. 
Quadro 3. Valores de área, perímetro, alongamento, arredondamento, diâmetro de Feret e compacidade, considerando o método de preparo e a profundidade de amostragem, e valores dos contrastes ortogonais para a classe de agregados de 0,50 a 0,21 mm

\begin{tabular}{|c|c|c|c|c|}
\hline \multirow{2}{*}{ Método de preparo } & \multicolumn{4}{|c|}{ Profundidade (m) } \\
\hline & 0,000 a 0,075 & 0,075 a 0,150 & 0,150 a 0,225 & 0,225 a 0,300 \\
\hline & \multicolumn{4}{|c|}{ Área, $\mathrm{mm}^{2}$} \\
\hline Plantio direto (PD) & 0,413 & 0,165 & 0,187 & 0,190 \\
\hline $\begin{array}{l}\text { Escarificador (E) } \\
\text { Grade }(G)\end{array}$ & $\begin{array}{l}0,178 \\
0,176\end{array}$ & $\begin{array}{l}0,170 \\
0,170\end{array}$ & $\begin{array}{l}0,167 \\
0,173\end{array}$ & $\begin{array}{l}0,154 \\
0,168\end{array}$ \\
\hline $\operatorname{Disco}(D)$ & 0,178 & 0,151 & 0,155 & 0,150 \\
\hline Aiveca (A) & 0,1744 & 0,162 & 0,172 & 0,144 \\
\hline $\mathrm{C} 1$ & $0,945 \#$ & ns & ns & $0,144^{\#}$ \\
\hline $\mathrm{C} 2$ & ns & ns & ns & ns \\
\hline C3 & ns & ns & ns & ns \\
\hline \multirow[t]{2}{*}{ C4 } & ns & ns & ns & ns \\
\hline & \multicolumn{4}{|c|}{ Perímetro, mm } \\
\hline Plantio direto (PD) & 1,535 & 1,632 & 1,734 & 1,738 \\
\hline $\begin{array}{l}\text { Escarificador (E) } \\
\text { Grade }(G)\end{array}$ & $\begin{array}{l}1,685 \\
1,664\end{array}$ & $\begin{array}{l}1,635 \\
1649\end{array}$ & $\begin{array}{l}1,628 \\
1,719\end{array}$ & $\begin{array}{l}1,545 \\
1,658\end{array}$ \\
\hline $\operatorname{Disco}(\mathrm{D})$ & $\begin{array}{l}1,004 \\
1,705\end{array}$ & $\begin{array}{l}1,049 \\
1,573\end{array}$ & $\begin{array}{l}1,119 \\
1,571\end{array}$ & $\begin{array}{l}1,038 \\
1,554\end{array}$ \\
\hline Aiveca (Á) & 1,673 & 1,606 & 1,657 & 1,497 \\
\hline C1 & ns & ns & ns & $0,698^{\#}$ \\
\hline C2 & ns & ns & ns & ns \\
\hline \multirow{3}{*}{ C4 } & ns & ns & ns & ns \\
\hline & ns & ns & ns & ns \\
\hline & \multicolumn{4}{|c|}{ Alongamento } \\
\hline Plantio direto (PD) & 0,723 & 0,710 & 0,720 & 0,719 \\
\hline Escarificador (E) & 0,720 & 0,721 & 0,727 & 0,724 \\
\hline Grade (G) & 0,723 & 0,714 & 0,699 & 0,714 \\
\hline Disco (D) & 0,720 & 0,711 & 0,713 & 0,715 \\
\hline Aiveca $(A)$ & 0,703 & 0,708 & 0,714 & 0,720 \\
\hline C1 & ns & ns & $0,028^{\#}$ & ns \\
\hline$C 2$ & ns & $0,030 \#$ & $0,054 * *$ & ns \\
\hline C3 & ns & ns & $-0,028^{*}$ & ns \\
\hline \multirow[t]{2}{*}{ C4 } & ns & ns & ns & ns \\
\hline & \multicolumn{4}{|c|}{ Arredondamento } \\
\hline Plantio direto (PD) & 0,698 & 0,775 & 0,773 & 0,780 \\
\hline Escarificador (E) & 0,779 & 0,789 & 0,784 & 0,803 \\
\hline Grade (G) & 0,786 & 0,776 & 0,736 & 0,769 \\
\hline Disco (D) & 0,768 & 0,762 & 0,782 & 0,777 \\
\hline Aiveca (A) & $\begin{array}{l}0,773 \\
-0,315 *\end{array}$ & 0,784 & 0,778 & 0,798 \\
\hline $\begin{array}{l}\text { C1 } \\
\text { C2 }\end{array}$ & $\begin{array}{c}-0,315^{* *} \\
\text { ns }\end{array}$ & $\begin{array}{l}\text { ns } \\
\text { ns }\end{array}$ & ns & ns \\
\hline \multirow{3}{*}{ C4 } & $\begin{array}{l}\text { ns } \\
\text { ns }\end{array}$ & $\begin{array}{l}\text { ns } \\
\text { ns }\end{array}$ & $\begin{array}{l}\text { ns } \\
-0,086^{*}\end{array}$ & $\begin{array}{l}\text { ns } \\
\text { ns }\end{array}$ \\
\hline & & ns & ns & ns \\
\hline & \multicolumn{4}{|c|}{ Diâmetro de Feret, mm } \\
\hline Plantio direto (PD) & 0,538 & 0,449 & 0,477 & 0,480 \\
\hline Escarificador (E) & 0,464 & 0,455 & $\begin{array}{l}0,449 \\
0,457\end{array}$ & 0,433 \\
\hline $\operatorname{Disco}(\mathrm{D})$ & 0,466 & 0,428 & 0,433 & 0,427 \\
\hline Aiveca (A) & 0,460 & 0,444 & 0,455 & 0,418 \\
\hline $\begin{array}{l}\mathrm{C} 1 \\
\mathrm{C}^{2}\end{array}$ & $0,301^{*}$ & $\begin{array}{l}\text { ns } \\
\text { ns }\end{array}$ & ns & $0,191^{\#}$ \\
\hline $\mathrm{C}^{2}$ & $\begin{array}{l}\text { ns } \\
\text { ns }\end{array}$ & ns & ns & ns \\
\hline \multirow[t]{2}{*}{ C4 } & $\begin{array}{l}\text { ns } \\
\text { ns }\end{array}$ & $\begin{array}{l}\text { ns } \\
\text { ns }\end{array}$ & $\begin{array}{l}\text { ns } \\
\text { ns }\end{array}$ & $\begin{array}{l}\text { ns } \\
\text { ns }\end{array}$ \\
\hline & \multicolumn{4}{|c|}{ Compacidade } \\
\hline Plantio direto (PD) & 0,874 & & 0,863 & \\
\hline Escarificador (E) & 0,863 & 0,867 & 0,871 & 0,875 \\
\hline $\begin{array}{l}\text { Grade (G) } \\
\text { Disco (D) }\end{array}$ & $\begin{array}{l}0,8 / 0 \\
0,861\end{array}$ & $\begin{array}{l}0,861 \\
0,862\end{array}$ & 0,866 & 0,866 \\
\hline Aiveca (Á) & 0,856 & 0,860 & 0,863 & 0,876 \\
\hline C1 & $0,047 \#$ & ns & ns & $-0,025 \#$ \\
\hline C2 & ns & ns & $0,040^{*}$ & $0,023^{\#}$ \\
\hline C3 & $0,022^{\#}$ & ns & $-0,040 *$ & $-0,024 *$ \\
\hline & ns & ns & ns & $-0,009 *$ \\
\hline
\end{tabular}

$\mathrm{C} 1=4 \mathrm{PD}-(\mathrm{E}+\mathrm{G}+\mathrm{D}+\mathrm{A}) ; \mathrm{C} 2=3 \mathrm{E}-(\mathrm{G}+\mathrm{D}+\mathrm{A}) ; \mathrm{C} 3=2 \mathrm{G}-(\mathrm{D}+\mathrm{A}) ; \mathrm{C} 4=\mathrm{DA}$.

ns:não-significativo; ${ }^{*}, \mathrm{e}^{* *}$ : significativos a 20,5 e $1 \%$, respectivamente, pelo teste $\mathrm{F}$. 
Quadro 4. Valores de área, perímetro, alongamento, arredondamento, diâmetro de Feret e compacidade, considerando o método de preparo e a profundidade de amostragem, e valores dos contrastes ortogonais para a classe de agregados de 0,21 a 0,105 mm

\begin{tabular}{|c|c|c|c|c|}
\hline \multirow{2}{*}{ Método de preparo } & \multicolumn{4}{|c|}{ Profundidade (m) } \\
\hline & 0,000 a 0,075 & 0,075 a 0,150 & 0,150 a 0,225 & 0,225 a 0,300 \\
\hline & \multicolumn{4}{|c|}{ Área, mm² } \\
\hline $\begin{array}{l}\text { Plantio direto (PD) } \\
\text { Escarificador (E) } \\
\text { Grade (G) } \\
\text { Disco (D) } \\
\text { Aiveca (A) } \\
\text { C1 } \\
\text { C2 } \\
\text { C3 } \\
\text { C4 }\end{array}$ & $\begin{array}{l}0,036 \\
0,044 \\
0,043 \\
0,039 \\
0,040 \\
-0,024^{* *} \\
0,009^{\#} \\
0,007^{\#} \\
\text { ns }\end{array}$ & $\begin{array}{c}0,039 \\
0,046 \\
0,047 \\
0,042 \\
0,041 \\
-0,021^{*} \\
\text { ns } \\
0,011^{*} \\
\text { ns }\end{array}$ & $\begin{array}{l}0,040 \\
0,044 \\
0,044 \\
0,039 \\
0,042 \\
\text { ns } \\
\text { ns } \\
\text { ns } \\
\text { ns }\end{array}$ & $\begin{array}{l}0,043 \\
0,047 \\
0,044 \\
0,041 \\
0,043 \\
\text { ns } \\
0,012 \# \\
\text { ns } \\
\text { ns }\end{array}$ \\
\hline & \multicolumn{4}{|c|}{ Perímetro, mm } \\
\hline $\begin{array}{l}\text { Plantio direto (PD) } \\
\text { Escarificador (E) } \\
\text { Grade (G) } \\
\text { Disco (D) } \\
\text { Aiveca (A) } \\
\text { C1 } \\
\text { C2 } \\
\text { C3 } \\
\text { C4 }\end{array}$ & $\begin{array}{c}0,711 \\
0,807 \\
0,789 \\
0,746 \\
0,758 \\
-0,256^{* *} \\
0,129^{*} \\
0,073^{\#} \\
\text { ns }\end{array}$ & $\begin{array}{c}0,741 \\
0,818 \\
0,828 \\
0,778 \\
0,768 \\
-0,227 \# \\
\text { ns } \\
\text { ns } \\
\text { ns }\end{array}$ & $\begin{array}{c}0,768 \\
0,806 \\
0,795 \\
0,742 \\
0,773 \\
\text { ns } \\
\text { ns } \\
\text { ns } \\
\text { ns }\end{array}$ & $\begin{array}{l}0,790 \\
0,834 \\
0,801 \\
0,763 \\
0,783 \\
\text { ns } \\
0,155^{\#} \\
\text { ns } \\
\text { ns }\end{array}$ \\
\hline $\mathrm{C} 4$ & \multicolumn{4}{|c|}{ Alongamento } \\
\hline $\begin{array}{l}\text { Plantio direto (PD) } \\
\text { Escarificador (E) } \\
\text { Grade (G) } \\
\text { Disco (D) } \\
\text { Aiveca (A) } \\
\text { C1 } \\
\text { C2 } \\
\text { C3 } \\
\text { C4 }\end{array}$ & $\begin{array}{l}0,707 \\
0,715 \\
0,728 \\
0,721 \\
0,716 \\
-0,052^{* *} \\
\text { ns } \\
0,018^{\#} \\
\text { ns }\end{array}$ & $\begin{array}{l}0,717 \\
0,722 \\
0,724 \\
0,713 \\
0,715 \\
\text { ns } \\
\text { ns } \\
0,021 \# \\
\text { ns }\end{array}$ & $\begin{array}{c}0,724 \\
0,717 \\
0,722 \\
0,724 \\
0,726 \\
\text { ns } \\
\text { ns } \\
\text { ns } \\
\text { ns }\end{array}$ & $\begin{array}{l}0,718 \\
0,730 \\
0,726 \\
0,726 \\
0,734 \\
-0,0449 \# \\
\text { ns } \\
\text { ns } \\
\text { ns }\end{array}$ \\
\hline $\mathrm{C} 4$ & \multicolumn{4}{|c|}{ Arredondamento } \\
\hline $\begin{array}{l}\text { Plantio direto (PD) } \\
\text { Escarificador (E) } \\
\text { Grade (G) } \\
\text { Disco (D) } \\
\text { Aiveca (A) } \\
\text { C1 } \\
\text { C2 } \\
\text { C3 } \\
\text { C4 }\end{array}$ & $\begin{array}{l}0,865 \\
0,827 \\
0,857 \\
0,864 \\
0,864 \\
\text { ns } \\
-0,103^{* *} \\
\text { ns } \\
\text { ns }\end{array}$ & $\begin{array}{l}0,863 \\
0,844 \\
0,838 \\
0,852 \\
0,863 \\
0,055^{\#} \\
\quad \text { ns } \\
-0,037^{*} \\
\quad \text { ns }\end{array}$ & $\begin{array}{c}0,851 \\
0,840 \\
0,846 \\
0,867 \\
0,866 \\
\text { ns } \\
-0,059 \# \\
-0,041^{\#} \\
\text { ns }\end{array}$ & $\begin{array}{l}0,848 \\
0,830 \\
0,858 \\
0,866 \\
0,873 \\
\text { ns } \\
-0,105^{* *} \\
\text { ns } \\
\text { ns }\end{array}$ \\
\hline C4 & \multicolumn{4}{|c|}{ Diâmetro de Feret, mm } \\
\hline $\begin{array}{l}\text { Plantio direto (PD) } \\
\text { Escarifi cador (E) } \\
\text { Grade (G) } \\
\text { Disco (D) } \\
\text { Aiveca (A) } \\
\text { C1 } \\
\text { C2 } \\
\text { C3 } \\
\text { C4 }\end{array}$ & $\begin{array}{l}0,207 \\
0,230 \\
0,229 \\
0,218 \\
0,2215 \\
-0,070^{* *} \\
0,021^{\#} \\
0,018^{\#} \\
\text { ns }\end{array}$ & $\begin{array}{c}0,216 \\
0,235 \\
0,238 \\
0,225 \\
0,224 \\
-0,058^{*} \\
\text { ns } \\
0,026^{*} \\
\text { ns }\end{array}$ & $\begin{array}{c}0,221 \\
0,232 \\
0,229 \\
0,217 \\
0,226 \\
\text { ns } \\
\text { ns } \\
\text { ns } \\
\text { ns }\end{array}$ & $\begin{array}{l}0,228 \\
0,238 \\
0,233 \\
0,223 \\
0,230 \\
\text { ns } \\
\text { ns } \\
\text { ns } \\
\text { ns }\end{array}$ \\
\hline & \multicolumn{4}{|c|}{ Compacidade } \\
\hline $\begin{array}{l}\text { Plantio direto (PD) } \\
\text { Escarificador (E) } \\
\text { Grade (G) } \\
\text { Disco (D) } \\
\text { Aiveca (A) } \\
\text { C1 } \\
\text { C2 } \\
\text { C3 } \\
\text { C4 }\end{array}$ & $\begin{array}{c}0,881 \\
0,879 \\
0,891 \\
0,890 \\
0,886 \\
-0,021^{\#} \\
-0,030^{*} \\
\text { ns } \\
\text { ns }\end{array}$ & $\begin{array}{l}0,888 \\
0,884 \\
0,885 \\
0,880 \\
0,884 \\
\text { ns } \\
\text { ns } \\
\text { ns } \\
\text { ns }\end{array}$ & $\begin{array}{c}0,890 \\
0,881 \\
0,886 \\
0,893 \\
0,891 \\
\text { ns } \\
\text { ns } \\
-0,025^{\#} \\
\text { ns }\end{array}$ & $\begin{array}{l}0,883 \\
0,886 \\
0,889 \\
0,891 \\
0,894 \\
\text { ns } \\
\text { ns } \\
\text { ns } \\
\text { ns }\end{array}$ \\
\hline
\end{tabular}

$\mathrm{C} 1=4 \mathrm{PD}-(\mathrm{E}+\mathrm{G}+\mathrm{D}+\mathrm{A}) ; \mathrm{C} 2=3 \mathrm{E}-(\mathrm{G}+\mathrm{D}+\mathrm{A}) ; \mathrm{C} 3=2 \mathrm{G}-(\mathrm{D}+\mathrm{A}) ; \mathrm{C} 4=\mathrm{DA}$.

ns:não-significativo; ${ }^{*}, \mathrm{e}^{* *}$ : significativos a 20,5 e $1 \%$, respectivamente, pelo teste $\mathrm{F}$. 


\section{CONCLUSÕES}

1. A análise de imagens mostrou-se sensível à detecção de mudanças na morfologia dos agregados do solo, sendo bastante promissora como uma nova ferramenta nos estudos da estrutura do solo.

2. Utilizados por três anos consecutivos, os sistemas de manejo testados não promoveram diferenças morfológicas dos agregados do Latossolo Vermel ho distrófico típico textura argilosa.

\section{LITE RATURA CITADA}

BREWER, R. Fabric and mineral analysis of soils. New York, Robert Krieger, 1976. 173p.
DADALTO, G.G.; COSTA, L.M. \& MOURA FILHO, W. Alterações em características físicas de sol os cultivados com pastagem. R. Ceres, 36:317-329, 1989.

FERNANDES, M.R. Alterações em propriedades de um Latossolo Vermel ho-Amarelo distrófico, fase cerrado, decorrentes da modalidade de uso e manejo. Viçosa, Universidade Federal de Viçosa, 1982. 65p. (Tese de Mestrado)

RIBEIRO J UNIOR, J .I. Análises estatísticas no SAEG, Viçosa, Universidade Federal de Viçosa, 2001. 301p.

SUGUIO, K. Introdução à sedimentologia. São Paulo, Edgard Blucher, 1973. 317p.

WILCOX, C.D.; DOVE, S.B.; MCDAVID, W.D. \& GREER, D.B. UTHSCSA image tool: help on-line. San Antonio, Texas, Universidade de San Antonio,1997. não paginado. 
N. OLSZEVSKI et al. 